\title{
Magnetization-transfer ${ }^{31} \mathrm{P}$ NMR of biochemical exchange in vivo: Application to creatine kinase kinetics
}

\author{
Harald E. Möller ${ }^{\mathrm{a}, *}$ and Dirk Wiedermann ${ }^{\mathrm{b}}$ \\ ${ }^{a}$ Max-Planck-Institut für neuropsychologische Forschung, Stephanstraße 1a, D-04103 Leipzig, \\ Germany \\ ${ }^{\mathrm{b}}$ Max-Planck-Institut für neurologische Forschung, Gleueler Straße 50, D-50866 Köln, Germany
}

\begin{abstract}
Phosphorus-31 saturation-transfer NMR spectroscopy provides an elegant means to study fluxes through the creatine kinase reaction in human skeletal muscle. To obtain reliable quantitative kinetic information, experimental imperfections, such as incomplete saturation and radiofrequency bleed over need to be addressed appropriately. In resting muscle, creatine kinase was near equilibrium both in normal controls and in a patient with impaired oxidative phosphorylation. Oral intake of high doses of creatine monohydrate for several days resulted in significantly increased concentrations of phosphocreatine but had no measurable effect on the phosphocreatine resynthesis rate in resting muscle.
\end{abstract}

\section{Abbreviations}

$\mathrm{ADP}=$ adenosine $5^{\prime}$-diphosphate; ATP $=$ adenosine $5^{\prime}$-triphosphate $\mathrm{CK}=$ creatine kinase $\mathrm{Cr}=$ creatine; MEALS = mitochondrial myopathy, encephalopathy, lactic acidosis, and stroke-like episodes; $\mathrm{NMR}=$ nuclear magnetic resonance; $\mathrm{PCr}=$ phosphocreatine; $\mathrm{PDE}=$ phosphodiesters; $\mathrm{P}_{\mathrm{i}}=$ inorganic phosphate; $\mathrm{PME}=$ phosphomonoesters; $\mathrm{PP}=$ phosphorylation potential; $\mathrm{RF}=$ radiofrequency; $\mathrm{ST}=$ saturation transfer.

\section{Introduction}

Localized in vivo ${ }^{31} \mathrm{P}$ nuclear magnetic resonance (NMR) spectroscopy provides accurate information on relative concentrations of principal components of cellular bioenergetics, including adenosine $5^{\prime}$-triphosphate (ATP), phosphocreatine ( $\left.\mathrm{PCr}\right)$, and inorganic orthophosphate $\left(\mathrm{P}_{\mathrm{i}}\right)$. As opposed to traditional techniques in analytical biochemistry, NMR is entirely noninvasive and selectively sensitive to the unbound forms of cellular metabolites in the cytosolic compartment as only highly mobile compounds have sufficiently long transverse relaxation times, $T_{2}$, to give rise to sharp, well-resolved resonance lines. It is worth stressing that concentrations of the free metabolites are required for estimations of thermodynamic values and the assessment of metabolic regulation of the cellular energy status. Besides providing access to static concentrations of bioenergetic key metabolites, ${ }^{31} \mathrm{P}$ NMR holds also great potential for investigating metabolic turnover rates. A representative example of such dynamic applications used to study enzyme kinetics in the resting skeletal muscle will be discussed in more detail below.

\footnotetext{
${ }^{*}$ Corresponding author. Tel.: +49 3419940 212; Fax: +49 3419940 221; E-mail: moeller@cns.mpg.de.
} 


\section{Biochemical considerations}

The free energy liberated during the ATPase reaction, that is, the hydrolysis of ATP to adenosine $5^{\prime}$-diphosphate (ADP), is the primary energy source of the living cell:

$$
\mathrm{MgATP}^{2-}+\mathrm{H}_{2} \mathrm{O} \rightleftarrows \mathrm{MgADP}^{-}+\mathrm{H}_{2} \mathrm{PO}_{4}^{-}
$$

In tissues with high energy demand, such as skeletal muscle, heart muscle, or brain, ATP is buffered against depletion via a second reservoir of high-energy bonds in the form of $\mathrm{PCr}$, which is utilized by the creatine kinase $(\mathrm{CK})$ reaction to recycle ADP rapidly back to ATP:

$$
\mathrm{PCr}^{2-}+\mathrm{MgADP}^{-}+\mathrm{H}^{+} \underset{k_{\text {rev }}}{\stackrel{k_{\text {for }}}{\rightleftarrows}} \mathrm{Cr}+\mathrm{MgATP}^{2-} .
$$

Because the $\mathrm{CK}$ reaction is near equilibrium at rest,

$$
\frac{[\mathrm{Cr}][\mathrm{ATP}]}{[\mathrm{PCr}][\mathrm{ADP}]\left[\mathrm{H}^{+}\right]}=\frac{k_{\mathrm{for}}}{k_{\mathrm{rev}}}=K_{\mathrm{eq}},
$$

where, for simplicity, we have omitted negative charges and complexation of the adenosine phosphates in the notations of concentrations. In Eqs (2) and (3), $\mathrm{Cr}$ denotes free creatine. At $38^{\circ} \mathrm{C}$, a pH of 7, an ionic strength of 0.2 , and a concentration of free intracellular magnesium ions, $\left[\mathrm{Mg}^{2+}\right]=1 \mathrm{mmol} / \mathrm{l}$, the equilibrium constant is $K_{\text {eq }}=1.66 \times 10^{9} \mathrm{l} / \mathrm{mol}$ [1]. Near equilibrium, the velocities of the forward and reverse fluxes should be equal:

$$
\begin{aligned}
v_{\mathrm{for}} & =k_{+1}[\mathrm{PCr}] \\
& =k_{-1}[\mathrm{ATP}]=v_{\mathrm{rev}} .
\end{aligned}
$$

In Eq. (4), $k_{+1}=k_{\text {for }} \times[\mathrm{ADP}] \times\left[\mathrm{H}^{+}\right]$and $k_{-1}=k_{\text {rev }} \times[\mathrm{Cr}]$ represent pseudo-unimolecular rate constants.

A well-defined regulator of the mitochondrial ATP synthesis rate, $Q$, is ADP [2]. Being micromolar, the concentration of free ADP is too low to be directly assessable by in vivo ${ }^{31} \mathrm{P}$ NMR. However, cytosolic levels can be calculated from the CK equilibrium, Eq. (3), by making use of the $\left[\mathrm{P}_{\mathrm{i}}\right] /[\mathrm{PCr}]$ ratio [3] and the intracellular $\mathrm{pH}$, which is easily determined from the chemical-shift difference between the $\mathrm{P}_{\mathrm{i}}$ and $\mathrm{PCr}$ resonances [4]. Besides assessing unbound ADP, other parameters of the functional mitochondrial status that are obtained from ${ }^{31} \mathrm{P}$ NMR spectra include the phosphorylation potential (PP) and the ratio of the mitochondrial ATP synthesis rate over its maximum value, $Q / Q_{\max }=1 /\left\{1+\left(0.53 \times[\mathrm{PCr}] /\left[\mathrm{P}_{\mathrm{i}}\right]\right)\right\}[3]$. The phosphorylation potential, $\mathrm{PP}=[\mathrm{ATP}] /\left([\mathrm{ADP}] \times\left[\mathrm{P}_{\mathrm{i}}\right]\right)$, is directly related to the free energy of ATP hydrolysis [2]. Finally, the concentration of free intracellular $\mathrm{Mg}^{2+}$ is derived from the chemical-shift difference between the $\alpha$ - and $\beta$-phosphorus of $\operatorname{ATP}(\alpha$-ATP, $\beta$-ATP) [5,6]. A summary of data extracted from ${ }^{31} \mathrm{P}$ NMR spectra of resting normal human skeletal muscle is given in Table 1.

Magnetization-transfer NMR is a means of measuring fluxes through certain enzyme-catalyzed reactions in vivo provided that separate resonances are observed and the exchange rates are of the order of 
Table 1

\begin{tabular}{|c|c|c|}
\hline Variable & Unit & Value \\
\hline$\overline{[\mathrm{PCr}]}$ & $\mathrm{mmol} / \mathrm{l}$ & $28.9 \pm 1.5$ \\
\hline$\left[\mathrm{P}_{\mathrm{i}}\right]$ & $\mathrm{mmol} / \mathrm{l}$ & $4.5 \pm 0.7$ \\
\hline [ADP] & $\mu \mathrm{mol} / \mathrm{l}$ & $8.9 \pm 1.3$ \\
\hline$\left[\mathrm{Mg}^{2+}\right]$ & $\mathrm{mmol} / \mathrm{l}$ & $0.9 \pm 0.3$ \\
\hline $\mathrm{pH}$ & & $7.04 \pm 0.02$ \\
\hline PP & $1 / \mathrm{mmol}$ & $204 \pm 64$ \\
\hline$Q / Q_{\max }$ & & $0.23 \pm 0.03$ \\
\hline
\end{tabular}

the spin-lattice relaxation rates [9]. Early examples include the adenylate kinase [10] and the ATPase reaction [11]. Flux through the CK reaction has been most extensively investigated by this approach including perfused organs [12,13], whole animals [14-16], and human subjects [7,17-22]. The following discussion will focus on experiments designed to study the CK reaction in resting human skeletal muscle.

\section{Theory}

The groundwork of magnetization-transfer experiments to study rates of chemical-exchange reactions was laid in the pioneering work of Forsén and Hoffman [23,24]. For simplicity, let us consider the following generalized two-site reaction depicted in Fig. 1. The variety of different approaches to magnetizationtransfer experiments may be classified according to the presence or absence of selective radiofrequency (RF) irradiation in the mixing period (Fig. 2), during which incoherent transfer of magnetization occurs. Instead of a comprehensive general treatment, which may be found, for example, in [9], our discussion is focused on two basic saturation-transfer (ST) experiments.

\subsection{Recovery after selective saturation of one resonance}

In ST experiments of this class (Fig. 2a), the recovery of resonances A and B is monitored after selective saturation of one of the spin pools. In the absence of exchange, $M_{z, \mathrm{~A}}$ and $M_{z, \mathrm{~B}}$ would relax exponentially towards their thermal equilibrium values, $M_{0, \mathrm{~A}}$ and $M_{0, \mathrm{~B}}$. From to the principle of microreversibility, it follows that

$$
k_{+1} M_{0, \mathrm{~A}}=k_{-1} M_{0, \mathrm{~B}} .
$$

Besides relaxation, magnetization is also transferred incoherently between A and B by the exchange process. Mathematically, the net effect is conveniently described by a set of modified Bloch equations [25]. If exchange of transverse magnetization can be neglected, the time evolution of $M_{z, \mathrm{~A}}$ and $M_{z, \mathrm{~B}}$ is given by:

$$
\frac{\mathrm{d} M_{z, \mathrm{~A}}}{\mathrm{~d} t}=\frac{M_{0, \mathrm{~A}}}{T_{1, \mathrm{~A}}}-\frac{M_{z, \mathrm{~A}}}{\tau_{1, \mathrm{~A}}}+k_{-1} M_{z, \mathrm{~B}},
$$




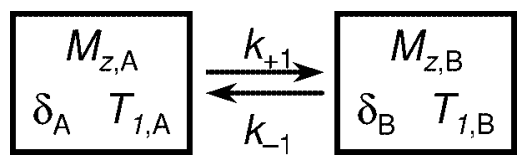

Fig. 1. Schematic of a two-site exchange system consisting of two pools of magnetization, $M_{z, \mathrm{~A}}$ and $M_{z, \mathrm{~B}}$, with chemical shifts $\delta_{\mathrm{A}}$ and $\delta_{\mathrm{B}}$, and longitudinal relaxation times, $T_{1, \mathrm{~A}}$ and $T_{1, \mathrm{~B}}$. Magnetization is transferred between both sites with forward and reverse rate constants $k_{\text {for }}$ and $k_{\text {rev }}$, respectively.

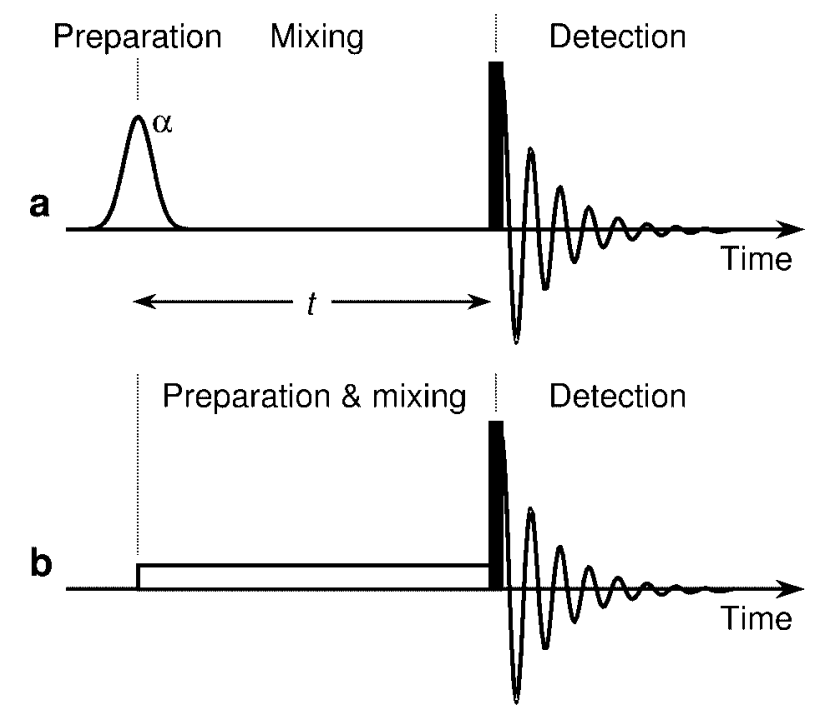

Fig. 2. Basic magnetization-transfer experiments: (a) class I without and (b) class II with selective irradiation in the mixing period between generation of non-equilibrium transverse magnetization (preparation) and readout of the NMR signal (detection). In the first case, an ideal pulse flip angle of $\alpha=180^{\circ}$ selectively inverts of the $z$-magnetization at site $\mathrm{B}$, consistent with the boundary condition $M_{z, \mathrm{~B}}(0)=-M_{0, \mathrm{~B}}$, whereas an ideal $90^{\circ}$ pulse leads to saturation of $\mathrm{B}$ and, hence, $M_{z, \mathrm{~B}}(0)=0$.

$$
\frac{\mathrm{d} M_{z, \mathrm{~B}}}{\mathrm{~d} t}=\frac{M_{0, \mathrm{~B}}}{T_{1, \mathrm{~B}}}-\frac{M_{z, \mathrm{~B}}}{\tau_{1, \mathrm{~B}}}+k_{+1} M_{z, \mathrm{~A}},
$$

where $\tau_{1, \mathrm{~A}}^{-1}=T_{1, \mathrm{~A}}^{-1}+k_{+1}$ and $\tau_{1, \mathrm{~B}}^{-1}=T_{1, \mathrm{~B}}^{-1}+k_{-1}$ are reciprocal lifetimes of the spin states at the respective sites. To avoid confusion, we note that $T_{1, \mathrm{~A}}$ and $T_{1, \mathrm{~B}}$ represent intrinsic spin-lattice relaxation times (i.e., if there were no exchange), which cannot be measured by conventional methods (e.g., inversion recovery). The generalized solutions to Eqs (5a) and (5b) are:

$$
\begin{aligned}
& M_{z, \mathrm{~A}}(t)=M_{0, \mathrm{~A}}+C_{1} \mathrm{e}^{-\lambda_{+} t}+C_{2} \mathrm{e}^{-\lambda_{-} t}, \\
& M_{z, \mathrm{~B}}(t)=M_{0, \mathrm{~B}}+C_{1}^{\prime} \mathrm{e}^{-\lambda_{+} t}+C_{2}^{\prime} \mathrm{e}^{-\lambda_{-} t}
\end{aligned}
$$

with $C_{1}^{\prime}=C_{1}\left(\tau_{1, \mathrm{~A}}^{-1}-\lambda_{+}\right) / k_{-1}$ and $C_{2}^{\prime}=C_{2}\left(\tau_{1, \mathrm{~A}}^{-1}-\lambda_{-}\right) / k_{-1}$. The coefficients $C_{1}$ and $C_{2}$ are obtained after inserting appropriate boundary conditions, such as $M_{z, \mathrm{~A}}(0)=M_{0, \mathrm{~A}}$ and $M_{z, \mathrm{~B}}(0)=0$ for instanta- 
neous selective saturation of the resonance at site B at $t=0$. The eigenvalues $\lambda_{+}$and $\lambda_{-}$are given by $\lambda_{ \pm}=\frac{1}{2}\left[\tau_{1, \mathrm{~A}}^{-1}+\tau_{1, \mathrm{~B}}^{-1} \pm \sqrt{\left(\tau_{1, \mathrm{~A}}^{-1}-\tau_{1, \mathrm{~B}}^{-1}\right)^{2} \pm 4 k_{+1} k_{-1}}\right]$. Assuming ideal ST leads to:

$$
\begin{aligned}
& M_{z, \mathrm{~A}}(t)=M_{0, \mathrm{~A}}\left[1+\frac{k_{+1}}{\lambda_{+}-\lambda_{-}}\left(\mathrm{e}^{-\lambda_{+} t}-\mathrm{e}^{-\lambda_{-} t}\right)\right], \\
& M_{z, \mathrm{~B}}(t)=M_{0, \mathrm{~B}}\left[1+\frac{\tau_{1, \mathrm{~A}}^{-1}-\lambda_{+}}{\lambda_{+}-\lambda_{-}} \mathrm{e}^{-\lambda_{+} t}-\frac{\tau_{1, \mathrm{~B}}^{-1}-\lambda_{-}}{\lambda_{+}-\lambda_{-}} \mathrm{e}^{-\lambda_{-} t}\right] .
\end{aligned}
$$

This result corresponds to biexponential recovery of the B resonance whereas a transient decrease of the A signal intensity is observed subsequent to the saturation of pool B.

\subsection{Relaxation in the presence of selective saturation of one resonance}

A simple experiment of this class (Fig. 2b), which is also referred to as time-dependent ST, is the observation of resonance A while pool B is selectively and completely saturated. Boundary conditions are thus $M_{z, \mathrm{~A}}(0)=M_{0, \mathrm{~A}}$ and $M_{z, \mathrm{~B}}(t)=0$ (i.e., $M_{z, \mathrm{~B}}$ drops out of Eq. (6a) being zero for all mixing times). As a result, $M_{z, \mathrm{~A}}$ decays according to

$$
M_{z, \mathrm{~A}}(t)=M_{0, \mathrm{~A}}\left[\frac{\tau_{1, \mathrm{~A}}}{T_{1, \mathrm{~A}}}+\left(1-\frac{\tau_{1, \mathrm{~A}}}{T_{1, \mathrm{~A}}}\right) \mathrm{e}^{-t / \tau_{1, \mathrm{~A}}}\right]
$$

approaching a new steady-state $M_{z, \mathrm{~A}}(\infty)=M_{0, \mathrm{~A}} /\left(1+k_{+1} T_{1, \mathrm{~A}}\right)$ in the limit of long mixing times, $t \rightarrow \infty$.

\subsection{Consideration of non-ideal RF irradiation}

In vivo ST studies, especially those involving human subjects, may be subject to errors resulting from experimental imperfections. Specifically, non-ideal situations may occur due to (i) incomplete saturation of pool B and (ii) imperfect selectivity of the saturation leading to unwanted partial irradiation at the A resonance (RF bleed over). To account for these effects in the theoretical analysis of our first class of ST experiments (Fig. 2a), appropriate boundary conditions are $M_{z, \mathrm{~B}}=\xi M_{0, \mathrm{~B}}$, where $\xi$ considers inhomogeneity of the irradiation ( $\xi=0$ for perfect saturation), and $M_{z, \mathrm{~A}}(0)=\zeta M_{0, \mathrm{~A}}$, where $\zeta$ considers RF bleed over (ideally, $\zeta=1$ ) [22]. Straightforward algebra assuming the simultaneous presence of both effects yields:

$$
\begin{aligned}
& M_{z, \mathrm{~A}}(t)=M_{0, \mathrm{~A}}[ 1+(1-\xi) \frac{k_{+1}\left(\mathrm{e}^{-\lambda_{+} t}-\mathrm{e}^{-\lambda_{-} t}\right)}{\lambda_{+}-\lambda_{-}} \\
&\left.+(1-\zeta)\left(\frac{\tau_{1, \mathrm{~A}}^{-1}-\lambda_{+}}{\lambda_{+}-\lambda_{-}} \mathrm{e}^{-\lambda_{+} t}-\frac{\tau_{1, \mathrm{~A}}^{-1}-\lambda_{-}}{\lambda_{+}-\lambda_{-}} \mathrm{e}^{-\lambda_{-} t}\right)\right], \\
& M_{z, \mathrm{~B}}(t)=M_{0, \mathrm{~B}}\left[1+(1-\xi)\left(\frac{\tau_{1, \mathrm{~A}}^{-1}-\lambda_{+}}{\lambda_{+}-\lambda_{-}} \mathrm{e}^{-\lambda_{+} t}-\frac{\tau_{1, \mathrm{~A}}^{-1}-\lambda_{-}}{\lambda_{+}-\lambda_{-}} \mathrm{e}^{-\lambda_{-} t}\right)\right. \\
&\left.+(1-\zeta) \frac{k_{-1}\left(\mathrm{e}^{-\lambda_{+} t}-\mathrm{e}^{-\lambda_{-} t}\right)}{\lambda_{+}-\lambda_{-}}\right] .
\end{aligned}
$$


Table 2

Apparent spin-lattice relaxation times (in seconds) of phosphate resonances measured at $1.5 \mathrm{~T}$ in normal human skeletal muscle without consideration of potential chemical-exchange effects [27]

\begin{tabular}{ccccccc}
\hline$\alpha$-ATP & $\beta$-ATP & $\gamma$-ATP & PCr & Pi & PME & PDE \\
\hline $2.8 \pm 0.3$ & $2.8 \pm 0.4$ & $3.4 \pm 0.1$ & $5.8 \pm 0.7$ & $3.9 \pm 0.4$ & $3.7 \pm 1.6$ & $3.1 \pm 1.9$ \\
\hline
\end{tabular}

An analysis of such imperfections present time-dependent ST experiments may be found elsewhere $[16,20]$.

\section{Experimental aspects}

Selective saturation of the resonance of either PCr or $\gamma$-ATP in investigations of the CK reaction is robustly achieved by either relatively long soft pulses or a DANTE (Delays Alternating with Nutations for Tailored Excitation) pulse train [26], which in the most simple case may precede an ordinary pulseand-acquire sequence. This approach can easily be combined with localization by a surface coil (i.e., the most commonly used experimental setup for studying human skeletal or heart muscle). As an example, a DANTE train of 25 hard pulses of 200- $\mu$ s duration and with 2-ms interpulse delays yielded an experimental excitation profile with a limited bandwith of approximately $15 \mathrm{~Hz}$ (full width at half maximum) and sidelobes at offset frequencies of $\pm n \times 500 \mathrm{~Hz}(n=1,2,3, \ldots)$ [22]. Ideally, the mixing time $t$ (Fig. 2a) should be varied in a step-wise fashion from well below to well above the spin-lattice relaxation times of the exchanging species for effectively tracing the state of the respective longitudinal magnetizations before and at equilibrium. Table 2 gives a summary of apparent in vivo ${ }^{31} \mathrm{P} T_{1}$ values (i.e., obtained without consideration of any chemical-exchange effects, see above).

Inhomogeneous RF irradiation leading to incomplete saturation of pool B (i.e., $\xi>0$ ) is easily detected in spectra recorded at minimal mixing times by the presence of residual signal intensity of the B resonance. Such imperfections were found to be relatively benign in numerical simulations leading to errors in $k_{ \pm 1}$ of order of $-10 \%$ if data analysis is performed assuming idealized conditions [22].

The $\gamma$-ATP resonance is shifted by $\Delta \delta=-2.4 \mathrm{ppm}$ (i.e., $\Delta \nu=-62 \mathrm{~Hz}$ at a magnetic field of $1.5 \mathrm{~T}$ ) from the PCr peak. Effects from unwanted off-resonance irradiation can be assessed by an additional single spectrum recorded with the transmitter frequency of the saturation pulse shifted from the PCr resonance symmetrically to higher values (i.e., centered at $+2.4 \mathrm{ppm}$ from $\mathrm{PCr}$, "mirror irradiation"). Any signal reduction of the $\mathrm{PCr}$ peak in this mirror-irradiation spectrum permits direct quantification of RF bleed over (i.e., $\zeta<1$ ). Numerical analysis demonstrated substantial overestimation of $k_{+1}$ (by more than $100 \%$ ) and underestimation of $k_{-1}$ (by more than 50\%) due to RF bleed over of only $20 \%$ if appropriate consideration of this source of systematic error is not included in the data analysis procedure [22].

\section{Application to human skeletal muscle}

An example of an in vivo ST experiment recorded in the calf muscle of a patient with an inborn mitochondrial defect (syndrome of mitochondrial myopathy, encephalopathy, lactic acidosis, and strokelike episodes, MELAS) that leads to impaired activities of complexes I or IV of the respiratory chain enzymes is presented in Fig. 3. The spectra recorded at the minimal mixing time ( $t=27 \mathrm{~ms})$ indicate that the DANTE pulse train used for selective irradiation achieved roughly $80 \%$ saturation at its center frequency. This deviation from perfect saturation (corresponding to $\xi \approx 0.20$ ) results from the inhomogeneous $\mathrm{RF}$ 


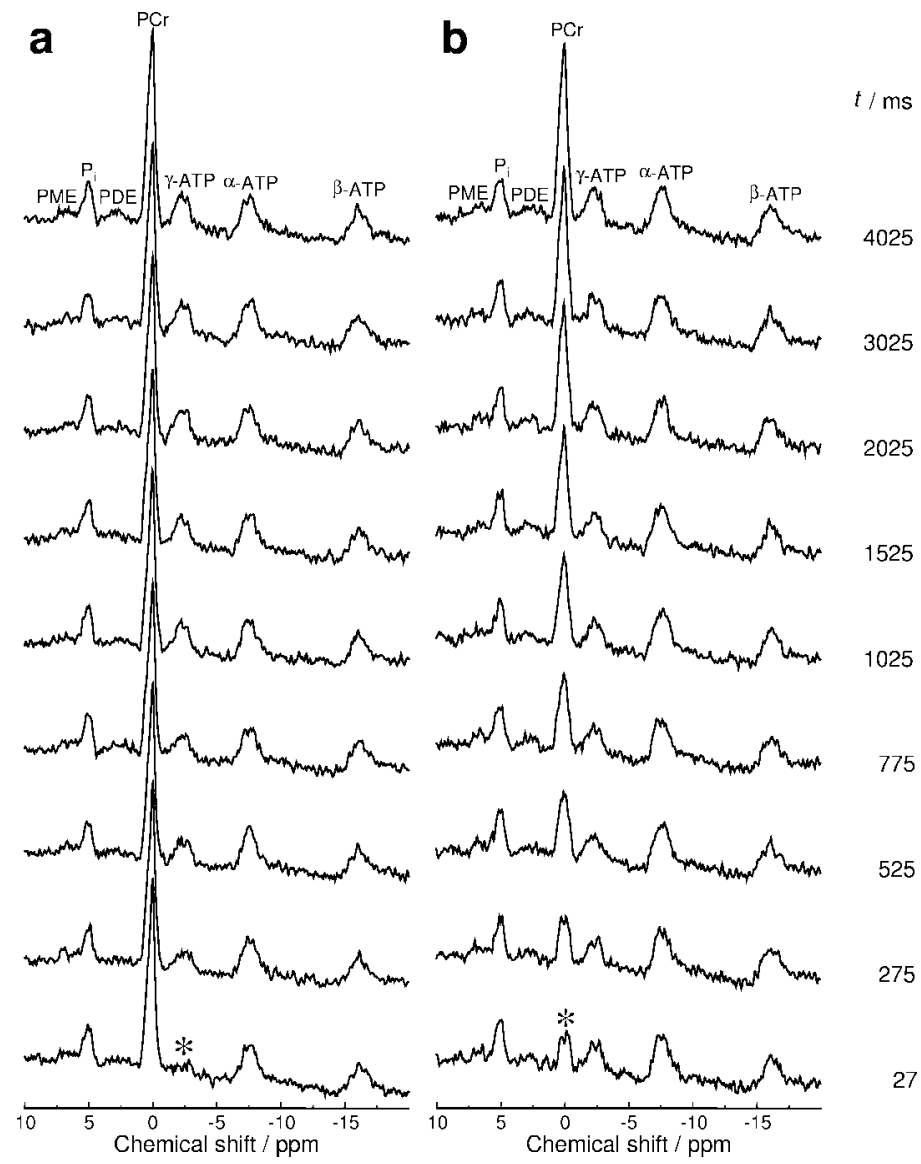

Fig. 3. Series of ${ }^{31} \mathrm{P}$ NMR spectra measured in vivo in resting skeletal muscle of a patient with defective mitochondrial function (MELAS). The assigned resonances include $\alpha-, \beta$-, and $\gamma$-ATP, PCr (chemical shift reference), $\mathrm{P}_{\mathrm{i}}$, phosphomonoesters (PME), and phosphodiesters (PDE). The center frequency of the DANTE pulse used for selective saturation of (a) $\gamma$-ATP or (b) PCr is marked by an asterisk. Both series of class-I ST experiments consists of nine spectra recorded at different mixing times $t$ (variation between 27 and $4025 \mathrm{~ms}$ ).

excitation profile of the 8-cm surface coil, which was used for both transmission and detection. An additional mirror-irradiation spectrum indicated significant RF bleed over leading to approximately $20 \%$ direct saturation (corresponding to $\zeta \approx 0.8$ ).

The experimentally observed variations of the PCr and $\gamma$-ATP peak areas with increasing mixing time are shown in Fig. 4 along with results from simultaneous non-linear least-squares fitting to Eqs (10a) and (10b). Relevant kinetic parameters characterizing the CK reaction in resting human skeletal muscle in both young, healthy volunteers and the patient are summarized in Table 3. Parameters describing flux through the forward CK reaction agree well with findings by other researchers, who did not assess the reverse reaction $[17,18]$. Consistent with animal studies, the ratio $k_{-1} / k_{+1}$ is roughly three $[14,15]$. Underlining that the $\mathrm{CK}$ reaction is near equilibrium, approximately identical fluxes are observed for the forward and the reverse reactions, $v_{\text {for }} \approx v_{\text {rev }}$. The most probable explanation of the subtle difference between both velocities, which is, however, insignificant is the fact that ATP is a substrate for a manifold of biochemical processes [13]. Consequently, the two-site model presented in Fig. 1 is likely to be an oversimplification, which works, however, sufficiently well within the achieved experimental accuracy. 

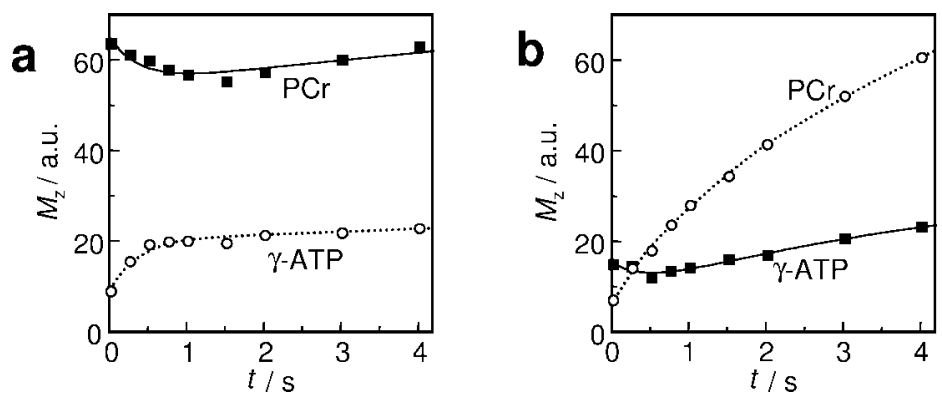

Fig. 4. Changes in the peak areas of PCr and $\gamma$-ATP (a.u. = arbitrary units) measured in a patient with MELAS and results from non-linear least-squares fitting (solid and dotted lines) to Eqs (10a) and (10b) for selective saturation of (a) $\gamma$-ATP and (b) PCr.

Table 3

Rate constants and fluxes through the $\mathrm{CK}$ reaction in resting human skeletal muscle measured using ${ }^{31}$ P ST NMR [7]

\begin{tabular}{lccc}
\hline Variable & Unit & Normal subjects & $\begin{array}{c}\text { Patient with } \\
\text { mitochondrial disorder }\end{array}$ \\
\hline$k_{+1}$ & $1 / \mathrm{s}$ & $0.39 \pm 0.13$ & 0.65 \\
$k_{-1}$ & $1 / \mathrm{s}$ & $0.99 \pm 0.09$ & 1.55 \\
$k_{-1} / k_{+1}$ & & $2.6 \pm 0.6$ & 2.4 \\
$v_{\text {for }}$ & $\mathrm{mmol} / \mathrm{l} / \mathrm{s}$ & $11.1 \pm 3.1$ & 15.0 \\
$v_{\text {rev }}$ & $\mathrm{mmol} / \mathrm{l} / \mathrm{s}$ & $8.1 \pm 0.7$ & 12.7 \\
\hline
\end{tabular}

The differences between the patient and control data in $k_{-1}$ and $v_{\text {rev }}$, both characterizing PCr resynthesis, were significant. Additional observations at static ${ }^{31} \mathrm{P}$ NMR spectroscopy of this patient included elevated [ADP] and [ $\left.\mathrm{P}_{\mathrm{i}}\right](17.0 \mu \mathrm{mol} / \mathrm{l}$ and $6.9 \mathrm{mmol} / \mathrm{l}$, respectively), reduced [PCr] $(23.0 \mathrm{mmol} / \mathrm{l})$, and increased $Q / Q_{\max }(0.38)$ [7]. Such abnormalities reflect the inability of the mitochondria to effectively utilize oxygen for ATP synthesis due to defective respiratory-chain enzymes and may be explained as the feedback consequence of mitochondrial function [2]. Elevated levels of ADP activate the CK reaction to restore ATP, thus interrelating ADP and PCr. One might speculate that the moderately increased $k_{+1}$ and the significantly increased $k_{-1}$ are due to increased [ADP] and free [Cr], respectively. However, as the CK reaction was found to be near equilibrium, concentration variations as observed in this patient are not likely to produce substantial changes in CK kinetics.

From both biochemical analyses of biopsy samples [28] and static ${ }^{31} \mathrm{P}$ NMR [29], it is well established that high-dose $\mathrm{Cr}$ intake for several days may elevate muscle $\mathrm{Cr}$ and $\mathrm{PCr}$ levels and improve performance during high-intensity, intermittent exercise [30]. Creatine supplementation has therefore become a popular ergogenic aid in sports [31] and was suggested for therapeutic use in MELAS [32]. An improved PCr resynthesis capacity was postulated as a means by which $\mathrm{Cr}$ loading might improve muscle performance during intermittent exercise bouts [31,32]. Static ${ }^{31} \mathrm{P}$ NMR spectroscopy of skeletal muscle following oral Cr loading yielded a significant increase in the PCr concentration by $+23 \%$ in normal subjects [22] and by $11 \%$ in the MELAS patient [7]. However, despite this rise in PCr, no indications of an improved PCr resynthesis capacity due to the $\mathrm{Cr}$ load were observed in ST ${ }^{31} \mathrm{P}$ NMR investigations of resting skeletal muscle CK fluxes. 


\section{Conclusion}

Saturation-transfer techniques are most useful for studying the kinetics of the CK reaction in the resting human skeletal muscle providing valuable dynamic information in addition to stationary concentration estimates from static spectra. However, reliable kinetic data are only obtained if experimental imperfections inherent in the pulse sequence, such as incomplete saturation and RF bleed over, are appropriately addressed. The rate of $\mathrm{PCr}$ resynthesis was near equilibrium at rest and, hence, not limited by the $\mathrm{CK}$ reaction. Consequently, increases in $\mathrm{PCr}$ (and assumable $\mathrm{Cr}$ ) following oral $\mathrm{Cr}$ supplementation have little effect, if any, on the $\mathrm{CK}$ reaction. These findings do, however, not necessarily exclude the possibility of changes in the PCr-ATP fluxes at higher levels of exercise or during recovery from maximal exercise.

\section{Acknowledgements}

We thank Thorsten Hilbich, Manfred Pützler, and Peter Vermathen for encouraging cooperation in previous studies of muscular metabolism, Albert Fromme, Joachim Schneider, and Lothar Thorwesten for support with the creatine loading experiments, and Gerhard Kurlemann for referral of the MELAS patient. Financial support by the Deutsche Forschungsgemeinschaft (Mo 588/3-1) is gratefully acknowledged.

\section{References}

[1] J.W. Lawson and R.L. Veech, Effects of $\mathrm{pH}$ and free $\mathrm{Mg}^{2+}$ on the $K_{e q}$ of the creatine kinase reaction and other phosphate hydrolyses and phosphate transfer reactions, J. Biol. Chem. 254 (1979), 6528-6537.

[2] G.J. Kemp, Interactions of mitochondrial ATP synthesis and the creatine kinase equilibrium in skeletal muscle, J. Theor. Biol. 170 (1994), 239-246.

[3] B. Chance, J.S. Leigh, B.J. Clark, J. Maris, J. Kent, S. Nioka and D. Smith, Control of oxidative metabolism and oxygen delivery in human skeletal muscle: A steady-state analysis of the work/energy cost transfer function, Proc. Natl. Acad. Sci. USA 82 (1985), 8384-8388.

[4] D.J. Taylor, P. Styles, P.M. Matthews, D.A. Arnold, D.G. Gadian, P. Bore and G.K. Radda, Energetics of human muscle: Exercise-induced ATP depletion, Magn. Reson. Med. 3 (1986), 44-54.

[5] R.K. Gupta, J.L. Benovic and Z.B. Rose, The determination of the free magnesium level in the human red blood cell by ${ }^{31}$ P NMR, J. Biol. Chem. 253 (1978), 6172-6176.

[6] G.D. Williams, T.J. Mosher and M.B. Smith, Simultaneous determination of intracellular magnesium and $\mathrm{pH}$ from the three ${ }^{31}$ P NMR chemical shifts of ATP, Anal. Biochem. 214 (1993), 458-467.

[7] H.E. Möller, D. Wiedermann, G. Kurlemann, T. Hilbich and G. Schuierer, Application of NMR spectroscopy to monitoring MELAS treatment: A case report, Muscle Nerve 25 (2002), 593-600.

[8] R.C. Harris, E. Hultman and L.O. Nordesjo, Glycogen, glycolytic intermediates and high-energy phosphates determined in biopsy samples of musculus quadriceps femoris of man at rest. Methods and variance of values, Scand. J. Clin. Lab. Invest. 33 (1974), 109-120.

[9] M. Rudin and A. Sauter, Measurement of reaction rates in vivo using magnetization transfer techniques, in: NMR Basic Principles and Progress, Vol. 27, P. Diehl, E. Fluck, H. Günther, R. Kosfeld and J. Seelig, eds, Springer, Berlin, 1992, pp. 257-293.

[10] T.R. Brown and S. Ogawa, ${ }^{31} \mathrm{P}$ nuclear magnetic resonance kinetic measurements on adenylate kinase, Proc. Natl. Acad. Sci. USA 74 (1977), 3627-3631.

[11] T.R. Brown, K. Ugurbil and R.G. Shulman, ${ }^{31} \mathrm{P}$ nuclear magnetic resonance measurements of ATPase kinetics in aerobic Escherichia coli cells, Proc. Natl. Acad. Sci. USA 74 (1977), 5551-5553.

[12] H. Degani, M. Laughlin, S. Campbell and R.G. Shulman, Kinetics of creatine kinase in heart: A ${ }^{31}$ P NMR saturation- and inversion-transfer study, Biochemistry 24 (1985), 5510-5516.

[13] K. Ugurbil, M. Petein, R. Maidan, S. Michurski and A.H.L. From, Measurement of an individual rate constant in the presence of multiple exchanges: Application to myocardial creatine kinase reaction, Biochemistry 25 (1986), 100-107. 
[14] J.A. Bittl, J. DeLayre and J.S. Ingwall, Rate equation for creatine kinase predicts the in vivo reaction velocity: ${ }^{31} \mathrm{P}$ NMR surface coil studies in brain, heart, and skeletal muscle of the living rat, Biochemistry 26 (1987), 6083-6090.

[15] P.S. Hsieh and R.S. Balaban, Saturation and inversion transfer studies of creatine kinase kinetics in rabbit skeletal muscle in vivo, Magn. Reson. Med. 7 (1988), 56-64.

[16] R.G.S. Spencer, A. Horská, J.A. Ferretti and G.H. Weiss, Spillover and incomplete saturation in kinetic measurements. J. Magn. Reson. B 101 (1993), 294-296.

[17] D. Rees, M.B. Smith, J. Harley and G.K. Radda, In vivo functioning of creatine phosphokinase in human forearm muscle, studied by ${ }^{31}$ P NMR saturation transfer, Magn. Reson. Med. 9 (1989), 39-52.

[18] J.F. Goudemant, M. Francaux, I. Mottet, R. Demeure, M. Sibomana and X. Sturbois, ${ }^{31}$ P NMR saturation transfer study of the creatine kinase reaction in human skeletal muscle at rest and during exercise, Magn. Reson. Med. 37 (1997), 744-753.

[19] W. Chen, X.H. Zhu, G. Adriany and K. Ugurbil, Increase of creatine kinase activity in the visual cortex of human brain during visual stimulation: A ${ }^{31}$ P NMR magnetization transfer study, Magn. Reson. Med. 38 (1997), 551-557.

[20] A. Horská and R.G.S. Spencer, Correctly accounting for radiofrequency spillover in saturation transfer experiments: Application to measurement of the creatine kinase reaction in human forearm muscle, MAGMA 5 (1997), 159-163.

[21] A. Horská, K.W. Fishbein, J.L. Fleg and R.G.S. Spencer, The relationship between creatine kinase kinetics and exercise intensity in human forearm is unchanged by age, Am. J. Physiol. Endocrinol. Metab. 279 (2000), E333-339.

[22] D. Wiedermann, J. Schneider, A. Fromme, L. Thorwesten and H.E. Möller, Creatine loading and resting muscle phosphocreatine flux: A saturation-transfer NMR study, MAGMA 13 (2001), 118-126.

[23] S. Forsén and R.A. Hoffman, Study of moderately rapid chemical exchange reactions by means of nuclear magnetic double resonance, J. Chem. Phys. 39 (1963), 2892-2901.

[24] R.A. Hoffman and S. Forsén, Transient and steady-state Overhauser experiments in the investigation of relaxation processes. Analogies between chemical exchange and relaxation, J. Chem. Phys. 45 (1966), 2049-2060.

[25] H.M. McConnell, Reaction rates by nuclear magnetic resonance, J. Chem. Phys. 28 (1958), 430-431.

[26] G.A. Morris and R. Freeman, Selective excitation in Fourier transform magnetic resonance, J. Magn. Reson. 29 (1978), 433-462.

[27] H.E. Möller, P. Vermathen, E. Rummeny, K. Wörtler, P. Wuisman, A. Rössner, B. Wörmann, J. Ritter and P.E. Peters, In vivo ${ }^{31} \mathrm{P}$ NMR spectroscopy of human musculoskeletal tumors as a measure of response to chemotherapy, NMR Biomed. 9 (1996), 347-358.

[28] R.C. Harris, K. Söderlund and E. Hultman, Elevation of creatine in resting and exercised muscle of normal subjects by creatine supplementation, Clin. Sci. (Colch) 83 (1992), 367-374.

[29] K. Vandenberghe, M. Goris, P. Van Hecke. M. Van Leemputte, L. Vangerven and P. Hespel, Long-term creatine intake is beneficial to muscle performance during resistance training, J. Appl. Physiol. 83 (1997), 2055-2063.

[30] P.L. Greenhaff, A. Casey, A.H. Short, R. Harris, K. Soderlund and E. Hultman, Influence of oral creatine supplementation of muscle torque during repeated bouts of maximal voluntary exercise in man, Clin. Sci. (Colch) 84 (1993), 565-571.

[31] P.D. Balsom, K. Söderlund and B. Ekblom, Creatine in humans with special reference to creatine supplementation, Sports Med. 18 (1994), 268-280.

[32] L. Hagenfeld, U. von Döbeln, G. Solders and L. Kaijser, Creatine treatment in MELAS, Muscle Nerve 17 (1994), 1236 1237. 


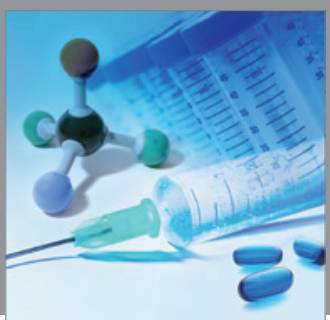

International Journal of

Medicinal Chemistry

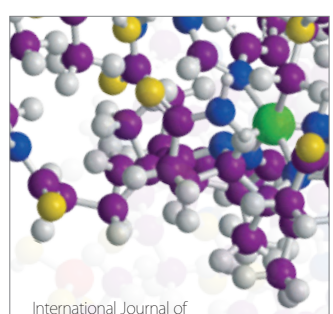

Carbohydrate Chemistry

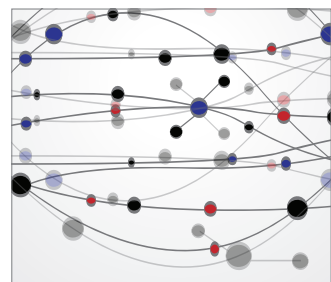

The Scientific World Journal
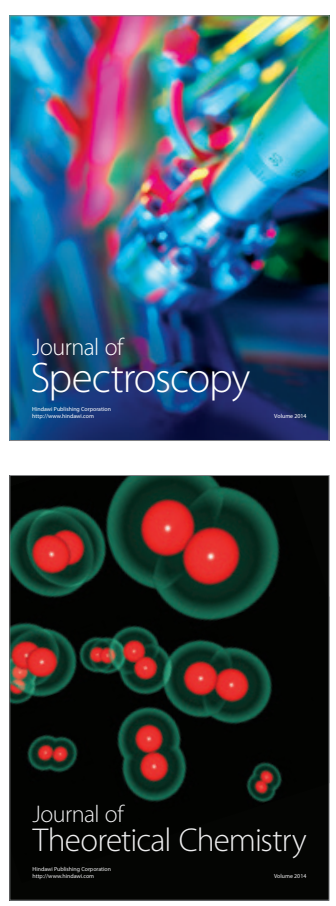
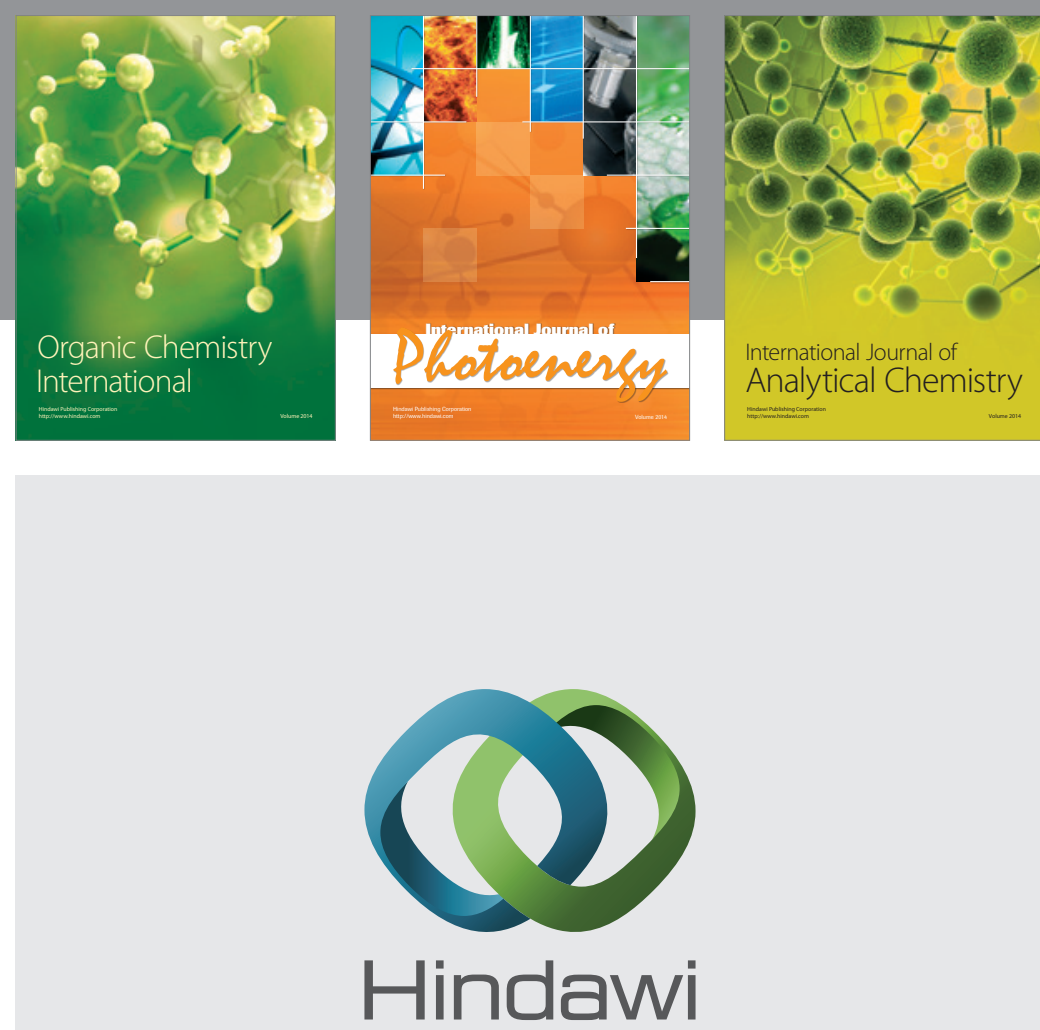

Submit your manuscripts at

http://www.hindawi.com
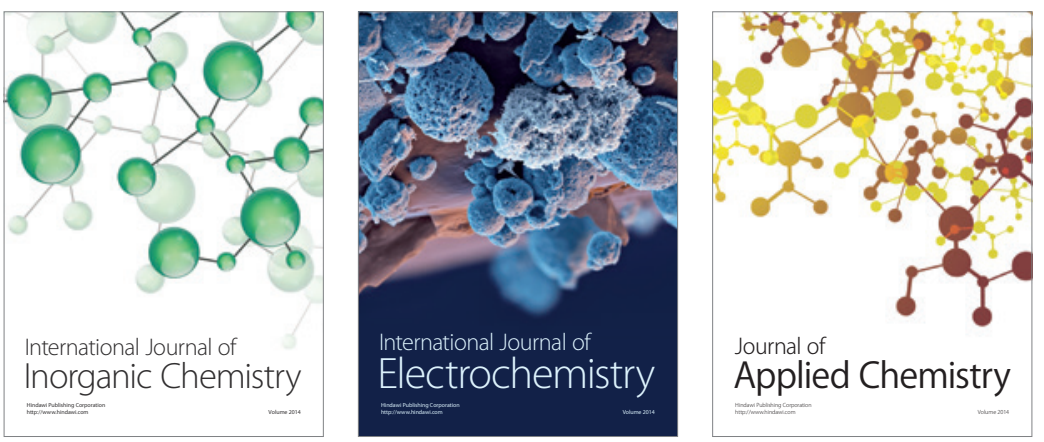

Journal of

Applied Chemistry
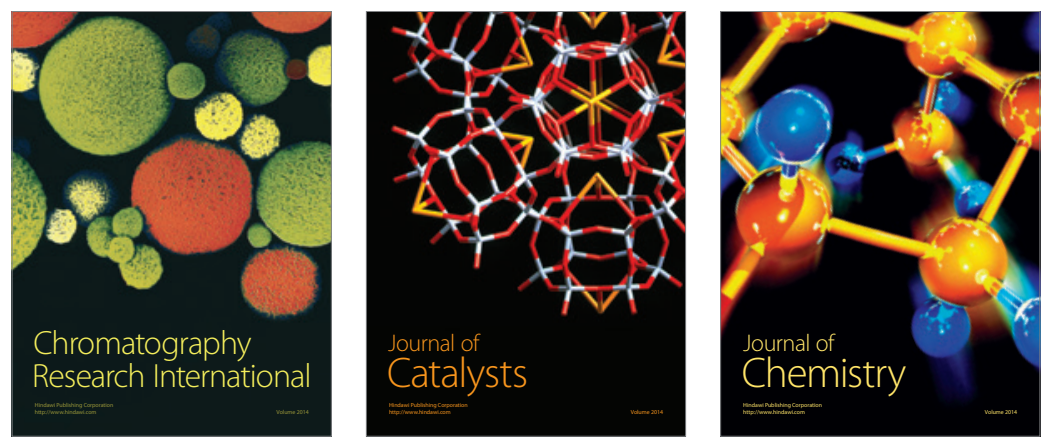
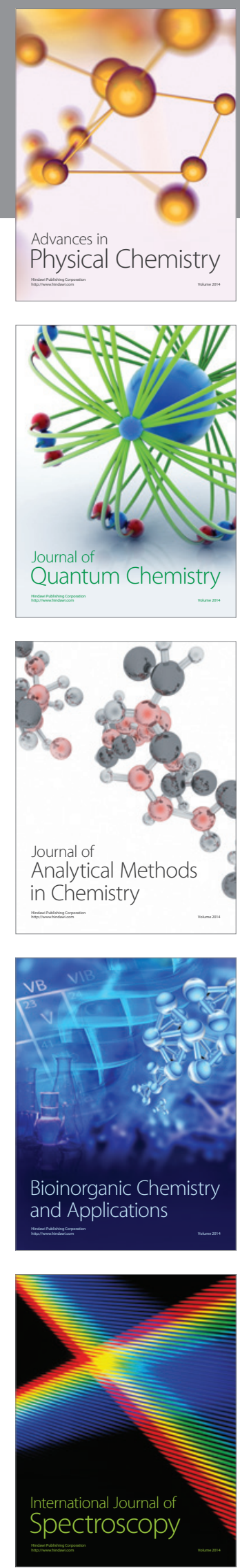